\title{
Pressure-Driven Thermal Slip Flow in the Elliptical Channel with Radial Oscillatory Wall
}

\author{
Nattawan Chuchalerm $\mathbb{D}^{1},{ }^{1}$ Benchawan Wiwatanapataphee $\mathbb{D}^{2},{ }^{2}$ and Wannika Sawangtong ${ }^{1,3}$ \\ ${ }^{1}$ Department of Mathematics, Faculty of Sciences, Mahidol University, Bangkok 10400, Thailand \\ ${ }^{2}$ School of Electrical Engineering, Computing and Mathematical Science, Curtin University, Perth, WA 6845, Australia \\ ${ }^{3}$ Centre of Excellence in Mathematics, Commission on Higher Education, Bangkok 10400, Thailand
}

Correspondence should be addressed to Nattawan Chuchalerm; nattawan.chc@student.mahidol.ac.th

Received 24 January 2020; Accepted 13 April 2020; Published 20 May 2020

Academic Editor: Qiankun Song

Copyright (C) 2020 Nattawan Chuchalerm et al. This is an open access article distributed under the Creative Commons Attribution License, which permits unrestricted use, distribution, and reproduction in any medium, provided the original work is properly cited.

\begin{abstract}
This paper is aimed at presenting thermal slip flow driven by oscillatory pressure gradient in a deformable microchannel of elliptic cross-section. The fully developed flow of Newtonian fluid is considered, and Navier slip is applied on the boundary. The boundary value problem is formulated and applied to the coronary blood flow-heat transfer phenomenon during thermotherapy treatment. Its semianalytical solutions of velocity and temperature fields are carried out by the Ritz method. The effects of oscillatory wall and slip length on velocity and temperature fields of blood are investigated.
\end{abstract}

\section{Introduction}

Experimental and numerical studies related to heat transfer and flow characteristics of fluid in an oscillating body are of great interest during the last few decades due to a wide range of engineering and physiological applications, namely, fuel pump in the aircraft fuel system, electric centrifugal pumps, pulsatile blood flow through large arteries, and gas exchange in human lung during high-frequency oscillatory ventilation.

Oscillatory flows of fluids have attracted several researchers in the literature. Experimentally, numerous devices have been set up for studying such flow driven by either a pressure drop or a volumetric pump. Some devices include a Starling resistor fixed between two rigid tubes and placed inside a pressure chamber [1] and a low-speed recirculating water channel with a hydrogen-bubble-generating device [2]. These studies conducted in experimental models at different operating conditions have provided invaluable information on the main characteristics of oscillatory flows such as drag reduction effects, wall-shear stress reduction, transitional Reynolds number, velocity timescales, and pressure fields.
Due to limitation of experimental models, a number of numerical investigations have been carried out with an aim to better understanding the effects of wall oscillation on the channel flow of a fluid. In literature, the governing equations of the oscillatory flow of fluid in the duct with oscillating wall are the continuity equation and the Navier-Stokes equations subject to either no-slip or slip boundary condition. Various studies considered two-dimensional oscillating flow in the duct with fully oscillating wall [3-9], partially oscillating wall $[10,11]$, and stretching sheet [12]. Three different directions of wall oscillation including the transverse (axial), vertical (radial), and both directions have been applied to the model.

For transverse oscillation of the wall, either fully or partially oscillatory wall has been investigated. Quadrio and Sibilla [3] applied a direct numerical simulation (DSN) to simulate turbulent flow in a pipe oscillating around the longitudinal axis. The effect of drag reduction was concerned due to the reference turbulent flows in a fixed pipe and a steadily rotating pipe. The maximum amount of drag reduction may be induced by the oscillating pipe. Xu and Huang [4] studied the impact of spanwise oscillating wall in the first 
two periods of oscillation on the turbulent plane channel flow at low Reynolds number using DSN. The periodic conditions in spanwise and streamwise directions were used, and no slip condition was imposed at the wall. Thomas et al. [5] applied a perturbation method to study the flow velocity and power requirements for fluid movement near oscillating wavy walls in transverse direction. It indicated that the more oscillating frequency of the wavy wall was, the faster the fluid oscillation and the more power requirements. Whittaker et al. [6] examined the energetic flow through a flexible tube in axial direction to determine the case of self-excited oscillations by investigating the effect of wall oscillation with varying a small amplitude and evaluating the energy budget.

In the case of partially transverse oscillation of the wall, Mateescu and Venditti [10] analysed the unsteady confined viscous flows with time variations of inflow velocities and partially transverse oscillation of the lower wall. The finite difference method was applied to the problem. They reported that the Reynolds number, oscillating frequency, and amplitude of the inflow velocity influenced on the formation of the flow separation regions. Yudhistira and Skote [11] presented a DSN to analyse the effect of a partially oscillating wall in the spanwise direction on the turbulent boundary layer. The flow characteristics including drag reduction and spatial development of drag reduction were related to the previous experiments.

The effects of wall oscillation in the radial direction have also been considered to analyse the drag reduction. Espin and Papageorgiou [7] investigated the stability of viscous pressure-driven flows in the channel with vertically oscillating walls and determined the numerical solutions for the Reynolds number ranges as an increase of wall oscillations amplitude. Shupti et al. [8] studied the two-dimensional pulsatile flow of blood through a stenosed artery which was assumed to be moving sinusoidally in the cross-section direction. The computational domain where a cosine-shaped aneurysm occurring after an appearance of a cosine-shaped stenosis causes the variation of the height of the domain. Blyth et al. [9] investigated the effect of wall amplitude and Reynolds number on an axisymmetric flow of a viscous incompressible fluid driven by a time-periodic wall motion of a cylindrical solid tube. By introducing a time-dependent stream function, the governing equations subject to the no-slip condition in the stream function vorticity form were solved in cylindrical polar coordinates. The results indicate that flow dynamics depend on the Reynolds number and the wall amplitude. A small or an intermediate value of the wall amplitude leads to quasiperiodic fields in time, and the flow field is time periodic at a large wall amplitude as an increase of Reynolds number. Adesanya and Makinde [13] investigated the effect of wall slip on twodimensional oscillatory flow of an incompressible viscous fluid through a channel of nonuniform wall temperature filled with a porous medium. In the last decade, heat transfer and fluid flow in the channel with oscillating wall have attracted too many researchers. Umuvathi et al. [14] determined the unsteady oscillatory flow and heat transfer in a horizontal channel filled with composite porous medium. Jha and Ajibade $[15,16]$ presented a free convective oscillatory flow of an incompressible viscous fluid between two periodically heated infinite vertical parallel plates and carried out some interesting results on the free convective oscillatory flows with given the time-dependent boundary conditions. AbdulHakeem and Sathiyanathan [17] derived analytical solutions of velocity and temperature fields for two-dimensional oscillatory flow of an incompressible viscous fluid on free convective radiation through a highly porous medium bounded by an infinite vertical plate. Sobh [18] used perturbation technique to study the peristaltic slip flow of viscoelastic fluid with heat and mass transfer in a uniform tube. The model is considered as the chyme movement in the small intestine. The analytic solutions show that the velocity, pressure gradient, temperature profile, and concentration field depend on the combined effect of slip parameter, heat, and mass transfer.

Various problems have been carried out to study the effects of slip flow and heat transfer in the channel but excluding the oscillating wall. Wiwatanapataphee et al. [19] studied transient oscillating pressure-driven slip flow and heat transfer in a microchannel of elliptic cross-section using the Ritz method. The results show the effects of the slip length and the aspect ratio of the microchannel on the velocity and temperature distribution. Mohyud-Din et al. [20] studied the effects of velocity and temperature slip of nanofluid in diverging and converging channels using variation iteration and variation of parameter methods. However, a few attempts have studied the pulsatile flow in the channel with wall oscillation in the radial direction, and most studies have ignored the slip condition, the oscillatory nature of the pressure gradient, and the heat transfer in the flow channel.

Recently, there has been considerable interest in developing sound and faithful thermal slip flow models that describe the coronary blood flow-heat transfer phenomenon during thermotherapy treatment. In the cancer treatment process, temperatures within a range of $42^{\circ} \mathrm{C}$ to $45^{\circ} \mathrm{C}$ can kill tumour cells without affecting normal tissue. As thermal therapy can cause improper blood flow and blood clots, understanding the complex phenomena of blood flow and heat transfer in a human coronary artery with oscillatory wall is important for the most efficacious treatment. This paper presents the driven pressure fluid flow and heat transfer in an elliptical channel with the oscillatory wall to predict the behaviour of circulatory flows inside the coronary arteries. Effects of oscillation amplitude and slip length on the velocity and temperature fields are investigated. The results are compared with existing ones available in the literature.

\section{Governing Equations}

In this section, we propose a mathematical model to study the behaviour of slip flow and heat transfer in an elliptical channel with the oscillating wall. Based on the assumption of fully developed flow, the problem is thus governed by the following boundary value problem (BVP) consisting of the system of the Navier-Stokes equations (1) and energy equation (2) subject to the initial condition (3) and the boundary conditions (4) and (5).

Navier-Stokes equation:

$$
\mu \nabla^{2} u^{*}-\rho \frac{\partial u^{*}}{\partial t}=\frac{\partial p}{\partial z}
$$


Energy equation:

$$
k \nabla^{2} T^{*}-\rho c_{p}\left(\frac{\partial T^{*}}{\partial t}+u^{*} \frac{\partial T^{*}}{\partial \mathrm{z}}\right)=q_{p} .
$$

Initial condition:

$$
T^{*}(0,0, z, 0)=T_{0} .
$$

Boundary conditions:

$$
\begin{array}{r}
u^{*}-u_{\mathrm{wall}}=-\beta \frac{\partial u^{*}}{\partial \mathbf{n}}, \\
h_{\infty}\left(T^{*}-T_{\infty}\right)=-k \frac{\partial T^{*}}{\partial \mathbf{n}},
\end{array}
$$

where $u^{*} \equiv u^{*}(t, x, y, z)$ and $T^{*} \equiv T^{*}(t, x, y, z)$ are, respectively, the velocity field and temperature field of fluid; $p$ is the pressure; $\rho, \mu, k$, and $c_{p}$ are, respectively, the density, viscosity, thermal conductivity, and specific heat of fluid; $q_{p}$ is the heat flux; $\beta$ and $u_{\text {wall }}$, represent, respectively, the slip length and tangential velocity of the wall; $h_{\infty}$ denotes the coefficient of convective heat transfer; and $T_{\infty}$ is the uniform external temperature.

We also consider no swirling flow with pressure variation in the flow direction [19], i.e.,

$$
\begin{aligned}
\frac{\partial u^{*}}{\partial z} & =0, \\
q_{p} & =\frac{q}{\bar{p}}\left(\frac{\partial p}{\partial t}+u^{*} \frac{\partial p}{\partial z}\right),
\end{aligned}
$$

where $\bar{p}$ is an average pressure.

In this study, the pressure gradient is prescribed by equation (8) based on the wall deformation, i.e.,

$$
\frac{\partial p}{\partial z}=-p_{0}\left[\left(a(t, \omega, \gamma)-a_{0}\right)+\left(b(t, \omega, \gamma)-b_{0}\right)\right]
$$

where $a(t, \omega, \gamma)$ and $b(t, \omega, \gamma)$ are semimajor and minor axes of the channel cross-section which are functions of frequency $(\omega)$, amplitude of oscillation $(\gamma)$, and time $(t)$, and $p_{0}, a_{0}$, and $b_{0}$ are constants.

Substituting equations (7) and (8) into the system of equations (1) and (2) yields the following BVP:

$$
\mu \nabla^{2} u-\rho \frac{\partial u}{\partial t}=-2 p_{0} \gamma \cos \omega t
$$

$k \nabla^{2} T^{*}-\rho c_{p}\left(\frac{\partial T^{*}}{\partial t}+u \frac{\partial T^{*}}{\partial z}\right)=\frac{2 q \gamma p_{0}}{\bar{p}}(z \omega \sin \omega t-u \cos \omega t)$,

subject to the initial condition (3), the boundary conditions (5), and

$$
u-u_{\text {wall }}+\beta \frac{\partial u}{\partial \mathbf{n}}=0 .
$$

In equation (9), $u \equiv u(t, x, y)$ is the function of $x, y$, and $t$. To determine the wall velocity $\left(u_{\text {wall }}\right)$, we consider the sinusoidal movement of the channel in the cross-stream direction. Let the variations in equation (11) of the semimajor and semiminor axes define, respectively, by

$$
\begin{aligned}
& a(t, \omega, \gamma)=a_{0}+\gamma \cos \omega t, \\
& b(t, \omega, \gamma)=b_{0}+\gamma \cos \omega t .
\end{aligned}
$$

By parametric equation of an ellipse, we have, on its perimeter,

$$
\begin{aligned}
& x=a(t, \omega, \gamma) \cos \psi, \\
& y=b(t, \omega, \gamma) \sin \psi,
\end{aligned}
$$

where $0 \leq \psi \leq 2 \pi$.

Thus, the wall velocity $u_{\text {wall }}$ can be determined by

$$
u_{\text {wall }}=\sqrt{\left(\frac{d x}{d t}\right)^{2}+\left(\frac{d y}{d t}\right)^{2}}=\gamma \omega \sin \omega t .
$$

\section{Ritz Method}

To apply the Ritz method for solving the above BVP (9), (10), (11), we firstly approximate the solution function of $u$ and $T^{*}$ in the following forms [19]:

$$
\begin{aligned}
u & \equiv u(t, x, y)=v(x, y) e^{i \omega t}, \\
T^{*} & \equiv T^{*}(t, x, y, z)=h(x, y)\left[T_{\infty}+\left(T_{0}-T_{\infty}\right) e^{-((\alpha z / \bar{u})+\lambda t)}\right],
\end{aligned}
$$

where $\bar{u} \equiv \bar{u}(t)$ is the mean velocity, $\alpha \equiv \alpha(t, \omega, \gamma)=4 h_{\infty} /$ $\left(\rho c_{p} D_{h}(t, \omega, \gamma)\right)$, and $\lambda \equiv \lambda(t, \omega, \gamma)=\sqrt{\left(h_{\infty} A(t, \omega, \gamma)\right) / c_{p}}$ in which $A(t, \omega, \gamma)$ is the cross-section area depending on $\omega, \gamma$, and $t$, and $D_{h}(t, \omega, \gamma)$ is a hydraulic diameter determined by

$$
D_{h}(t, \omega, \gamma)=\frac{4 a(t, \omega, \gamma) b(t, \omega, \gamma)\left(64-16 H^{2}\right)}{(a(t, \omega, \gamma)+b(t, \omega, \gamma))\left(64-3 H^{4}\right)},
$$

with

$$
H(t, \omega, \gamma)=\frac{a(t, \omega, \gamma)-b(t, \omega, \gamma)}{a(t, \omega, \gamma)+b(t, \omega, \gamma)} .
$$

In equation (15), $x$ and $y$ varies over time and we assume that rates of change of the $x$ and $y$ coordinates with respect to $t$ at the center $(x, y)=(0,0)$ are fixed, i.e., $d x_{0} / d t=d y_{0} / d t=0$ and the rates of change of the $x$ and $y$ coordinates with respect to $t$ on the boundary wall are defined by $d x_{w} / d t=-$ $\gamma \omega \sin \omega t \cos \psi$ and $d y_{w} / d t=-\gamma \omega \sin \omega t \sin \psi$. Then, the rates of change of the $x$ and $y$ coordinates with respect to $t$ in the fluid domain can be expressed as 


$$
\begin{aligned}
& \frac{d x}{d t}=\frac{(-\gamma \omega \sin \omega t \cos \psi) x}{a(t, \omega, \gamma) \cos \psi}, \\
& \frac{d x}{d t}=\frac{(-\gamma \omega \sin \omega t \sin \psi) y}{b(t, \omega, \gamma) \sin \psi} .
\end{aligned}
$$

From equation (15), we obtain

$$
\begin{aligned}
\frac{\partial u}{\partial t}= & v(x, y) i \omega e^{i \omega t}-\gamma \omega e^{i \omega t} \sin \omega t \\
& \cdot\left(\frac{x}{a(t, \omega, \gamma)} \frac{\partial v}{\partial x}+\frac{y}{b(t, \omega, \gamma)} \frac{\partial v}{\partial y}\right)
\end{aligned}
$$

In equation (16), two parameters including $\alpha=\alpha_{i}$ and $\lambda=\lambda_{i}$ are fixed. We then obtain

$$
\begin{aligned}
\frac{\partial T^{*}}{\partial t}= & \left(-\lambda+\frac{\alpha z}{\bar{u}^{2}} \frac{d \bar{u}}{d t}\right)\left(T_{0}-T_{\infty}\right) e^{-((\alpha / \bar{u}) z+\lambda t)} h(x, y) \\
& +\left(T_{\infty}+\left(T_{0}-T_{\infty}\right) e^{-((\alpha / \bar{u})+\lambda t)}\right) \frac{d h}{d t},
\end{aligned}
$$

where $d h / d t$ is the rate of change of thermal parameter.

Substituting equations (15), (16), (17), (18), (19), (21), (22) into equations (9) and (10), we obtain

$$
\begin{gathered}
\frac{\partial^{2} v}{\partial x^{2}}+\frac{\partial^{2} v}{\partial y^{2}}-\frac{\rho}{\mu}\left(i \omega v-\gamma \omega \sin \omega t\left(\frac{x}{a(t, \omega, \gamma)} \frac{\partial v}{\partial x}\right.\right. \\
\left.\left.+\frac{y}{b(t, \omega, \gamma)} \frac{\partial v}{\partial y}\right)\right)+\frac{2 \gamma p_{0} e^{-i \omega t} \cos \omega t}{\mu}=0, \\
\frac{\partial^{2} h}{\partial x^{2}}+\frac{\partial^{2} h}{\partial y^{2}}+g(t, x, y, z) h-f(t, x, y, z)=0,
\end{gathered}
$$

subject to initial condition $h(0,0, z, 0)=1$ and two boundary conditions

$$
\begin{aligned}
\frac{\partial v}{\partial \mathbf{n}} & =\frac{\gamma \omega e^{-i \omega t} \sin \omega t-v}{\beta}, \\
-k \frac{\partial h}{\partial \mathbf{n}} & =\frac{h_{\infty}\left(h-T_{\infty}\right)}{T_{\infty}+\left(T_{0}-T_{\infty}\right) e^{-((\alpha / \bar{u}) z+\lambda t)}} .
\end{aligned}
$$

In equation (24), $g(t, x, y, z)$ and $f(t, x, y, z)$ are defined by

$$
\begin{aligned}
& g(t, x, y, z)=\frac{\left[(\alpha / \bar{u})^{2}+\delta\left(\lambda-(\alpha z / \bar{u})^{2}(d \bar{u} / d t)+\alpha u / \bar{u}\right)\right]\left(T_{0}-T_{\infty}\right) e^{-((\alpha z / \bar{u})+\lambda t)}}{T_{\infty}+\left(T_{0}-T_{\infty}\right) e^{-((\alpha / \bar{u}) z+\lambda t)}}, \\
& f(t, x, y, z)=\frac{2 \gamma q p_{0}}{k \bar{p}}\left(\frac{z \omega \sin \omega t-u \cos \omega t}{T_{\infty}+\left(T_{0}-T_{\infty}\right) e^{-((\alpha z / \bar{u})+\lambda t)}}\right)+\delta \frac{d h}{d t},
\end{aligned}
$$

where $\delta=\rho c_{p} / k$.

To simplify the problem, we consider the flow at a crosssection $z=z_{n}$, and $t=t_{n}$ at instant time. We then solve the
BVP (23), (24), (26) for functions $v\left(t_{n}, x, y\right)$ and $h\left(t_{n}, x, y\right.$, $\left.z_{n}\right)$ by applying the quadratic minimization such that

$$
\min J=\frac{1}{2} B(w, w)-F,
$$

where $B(w, w)$ and $F$ are, respectively, bilinear and linear forms [21].

Hence, the BVP (23), (24), (26) is equivalent to the following system

$$
\begin{aligned}
I_{v}= & \frac{1}{\beta} \int_{\partial \Omega} v^{2}-v \gamma \omega e^{-i \omega t_{n}} \sin \omega t_{n} d s+\int_{\Omega}\left(\frac{\partial v}{\partial x}\right)^{2}+\left(\frac{\partial v}{\partial y}\right)^{2} \\
& +\frac{i \omega \rho v^{2}}{\mu}-\frac{v \rho \gamma \omega \sin \omega t_{n}}{\mu}\left(\frac{x}{a\left(t_{n}, \omega, \gamma\right)} \frac{\partial v}{\partial x}+\frac{y}{b\left(t_{n}, \omega, \gamma\right)} \frac{\partial v}{\partial y}\right) \\
& -\frac{4 v \gamma p_{0} e^{-i \omega t_{n}} \cos \omega t_{n}}{\mu} d \Omega=0,
\end{aligned}
$$

$$
\begin{aligned}
I_{h}= & \frac{h_{\infty}}{k} \int_{\partial \Omega} h^{2}-\frac{T_{\infty} h}{T_{\infty}+\left(T_{0}-T_{\infty}\right) e^{\left(-\alpha z_{n} / \bar{u}\right)-\lambda t_{n}}} d s+\int_{\Omega}\left(\frac{\partial h}{\partial x}\right)^{2} \\
& +\left(\frac{\partial h}{\partial y}\right)^{2}-g_{n}\left(t_{n}, x, y, z_{n}\right) h^{2}+2 f_{n}\left(t_{n}, x, y, z_{n}\right) h d \Omega=0,
\end{aligned}
$$

where

$$
\begin{gathered}
\Omega \equiv \Omega_{t}(x, y, \omega, \gamma)=\left\{(x, y) \mid \frac{x^{2}}{a^{2}\left(t_{n}, \omega, \gamma\right)}+\frac{y^{2}}{b^{2}\left(t_{n}, \omega, \gamma\right)} \leq 1\right\}, \\
\partial \Omega \equiv \partial \Omega_{t}(x, y, \omega, \gamma)=\left\{(x, y) \mid \frac{x^{2}}{a^{2}\left(t_{n}, \omega, \gamma\right)}+\frac{y^{2}}{b^{2}\left(t_{n}, \omega, \gamma\right)}=1\right\} .
\end{gathered}
$$

We now approximate functions $v(x, y)$ and $h(x, y)$ as a linear combination of $N$ known functions $\phi_{i}(x, y)$ and $\varphi_{i}$ $(x, y)$ as follows:

$$
\begin{aligned}
& v \equiv v(x, y)=\sum_{i=1}^{N} c_{i} \phi_{i}(x, y), \\
& h \equiv h(x, y)=1+\sum_{i=1}^{N} d_{i} \varphi_{i}(x, y),
\end{aligned}
$$

with

$$
\begin{aligned}
& \phi_{i}(x, y)=\left\{1, x^{2}, y^{2}, x^{4}, x^{2}, y^{2}, y^{4}, x^{2} y^{4}, x^{4} y^{2}, y^{6}, \cdots\right\} \\
& \varphi_{i}(x, y)=\left\{x y, x^{2}, y^{2}, x^{4}, x^{2}, y^{2}, y^{4}, x^{2} y^{4}, x^{4} y^{2}, y^{6}, \cdots\right\} .
\end{aligned}
$$

Substituting equation (32) into equation (29) and imposing $\partial I_{v} / \partial c_{i}=0(i=1, \cdots, N)$ yield the following linear system of equations: 


$$
\sum_{j=1}^{N} c_{j}\left(A_{i j}+A_{i j}^{b}\right)=C_{i}+C_{i}^{b}, \quad i=1, \cdots, N
$$

with

$$
\begin{aligned}
A_{i j}= & \int_{\Omega} \phi_{i x} \phi_{j x}+\phi_{i y} \phi_{j y}+\frac{i \omega \rho}{\mu} \phi_{i} \phi_{j}-\frac{\rho \gamma \omega \sin \omega t_{n}}{\mu} \\
& \cdot\left(\frac{x}{a\left(t_{n}, \omega, \gamma\right)} \phi_{i} \phi_{j x}+\frac{y}{b\left(t_{n}, \omega, \gamma\right)} \phi_{i} \phi_{j y}\right) d \Omega, \\
A_{i j}^{b}= & \frac{1}{\beta} \int_{\partial \Omega} \phi_{i} \phi_{j} d s, \\
C_{i}= & \frac{4 \gamma p_{0} e^{-i \omega t_{n}} \cos \omega t_{n}}{\mu} \int_{\Omega} \phi_{i} d \Omega \\
C_{i}^{b}= & \frac{\gamma \omega e^{-i \omega t_{n}} \sin \omega t_{n}}{\beta} \int_{\partial \Omega} \phi_{i} d s .
\end{aligned}
$$

Similarly, substituting equation (33) into equation (30) and setting $\partial I_{h} / \partial d_{i}=0(i=1, \cdots, N)$ give

$$
\sum_{j=1}^{N} d_{j}\left(B_{i j}+B_{i j}^{b}\right)=D_{i}+D_{i}^{b}, \quad i=1, \cdots, N
$$

with

$$
\begin{aligned}
B_{i j} & =\int_{\Omega} \varphi_{i x} \varphi_{j x}+\varphi_{i y} \varphi_{j y}-g_{n} \varphi_{i} \varphi_{j} d \Omega, \\
B_{i j}^{b} & =\frac{h_{\infty}}{k} \int_{\partial \Omega} \varphi_{i} \varphi_{j} d s, \\
D_{i} & =\int_{\Omega} 2 g_{n} \varphi_{i}-2 f_{n} \varphi_{i} d \Omega, \\
D_{i}^{b} & =-\frac{h_{\infty}}{k} \int_{\partial \Omega} 2 \varphi_{i}-\frac{T_{\infty} \varphi_{i}}{T_{\infty}+\left(T_{0}-T_{\infty}\right) e^{(-\alpha / \bar{u}) z_{n}-\lambda t_{n}}} d s .
\end{aligned}
$$

The problem is thus reduced to find the coefficients $c_{j}$ and $d_{j}$ by solving the following linear systems (35) and (37). Finally, the velocity and temperature fields are expressed by

$$
\begin{aligned}
u & \equiv \mathbb{R}\left(e^{i \omega t_{n}} \sum_{j=1}^{N} c_{j} \phi_{j}(x, y)\right), \\
T^{*} & =\left(1+\mathbb{R} \sum_{j=1}^{N} d_{j} \varphi_{j}(x, y)\right)\left(T_{\infty}+\left(T_{0}-T_{\infty}\right) e^{(-\alpha / \bar{u}) z_{n}-\lambda t_{n}}\right) .
\end{aligned}
$$

\section{Numerical Examples}

To describe the effect of wall deformation on the slip flows and heat transfer in the microchannel, we choose the computational domain of a coronary artery with the elliptical crosssection having the lengths of major semiaxis $a(t, \omega, \gamma)$ and
TABle 1: Computational domain.

\begin{tabular}{lccc}
\hline Parameter & Notation & Value & Unit \\
\hline Average major semiaxis & $a_{0}$ & 0.1 & $\mathrm{~cm}$ \\
Average minor semiaxis & $b_{0}$ & 0.075 & $\mathrm{~cm}$ \\
Amplitude of oscillation & $\gamma$ & {$[0.01,0.05]$} & $\mathrm{cm}$ \\
Frequency & $\omega$ & $\frac{2 \pi}{\tau}$ & \\
Period of radial & $\tau$ & 0.8 & $\mathrm{~s}$ \\
oscillation of channel & $\beta$ & {$[0.005,0.07]$} & \\
Slip length & & &
\end{tabular}

TABLE 2: Fluid properties [19].

\begin{tabular}{lccc}
\hline Parameter & Notation & Value & Unit \\
\hline Density & $\rho$ & 1.05 & $\mathrm{~g} / \mathrm{cm}^{3}$ \\
Dynamic viscosity & $\mu$ & 0.04 & Poise \\
Thermal conductivity & $k$ & $1.43 \times 10^{-3}$ & $\mathrm{cal} /\left(\mathrm{s} \cdot \mathrm{cm} \cdot{ }^{\circ} \mathrm{C}\right)$ \\
Specific heat capacity & $c_{p}$ & 0.86 & $\mathrm{cal} /\left(\mathrm{g} \cdot{ }^{\circ} \mathrm{C}\right)$ \\
Temperature & $T_{0}$ & 37 & ${ }^{\circ} \mathrm{C}$ \\
& $T_{\infty}$ & 42 & ${ }^{\circ} \mathrm{C}$ \\
Heat transfer coefficient & $h_{\infty}$ & $5.49 \times 10^{-5}$ & $\mathrm{cal} /\left(\mathrm{s} \cdot \mathrm{cm}^{2}\right.$. \\
Heat flux parameter & $q$ & 0.1 & $\mathrm{~W} / \mathrm{cm}^{2}$ \\
Average pressure & $\bar{p}$ & 100 & $\mathrm{mmHg}$ \\
Model parameter & $p_{0}$ & 40 & \\
Rate of change of thermal & $d h$ & $1 \times 10^{-3}$ & \\
parameter & $\frac{d t}{d t}$ & &
\end{tabular}

minor semiaxis $b(t, \omega, \gamma)$ as given in Table 1 . The average lengths of major/minor semiaxes depend on the variation of the hydraulic diameter $D_{h}$ of the ellipse. In this study, $D_{h}$ between $0.108 \mathrm{~cm}$ and $0.231 \mathrm{~cm}$ corresponding to the typical size of the coronary artery [22] and oscillation frequency and period corresponding to human heartbeat pattern are chosen. However, owing to the lack of data, we could not find realistic values of oscillation amplitude $\gamma$ and slip length $\beta$.

Heartbeat induces the oscillatory pressure gradient of blood and oscillating movement of the coronary arterial wall. To study oscillatory blood flow, blood is assumed to be an incompressible Newtonian fluid having the properties as shown in Table 2.

Figure 1 represents variations of radial wall and pressure gradient obtained from the model with wall oscillation for oscillation amplitude $\gamma=0.03 \mathrm{~cm}$ and frequency $\omega=5 \pi / 2$. It is noted that the wall is expanding during $d p / d z<0$, and the wall is contracting during $d p / d z>0$. At the time in which the highest negative pressure gradient exists $(d p / d z=-2.4)$, expansion of major/minor axes is the biggest, i.e., $a=0.13$ $\mathrm{cm}$ and $b=0.105 \mathrm{~cm}$. On the other hand, at the time in which the highest positive pressure gradient exists $(d p / d z=2.4)$, contraction of major $/$ minor axes is the smallest, i.e., $a=$ $0.07 \mathrm{~cm}$ and $b=0.045 \mathrm{~cm}$. 


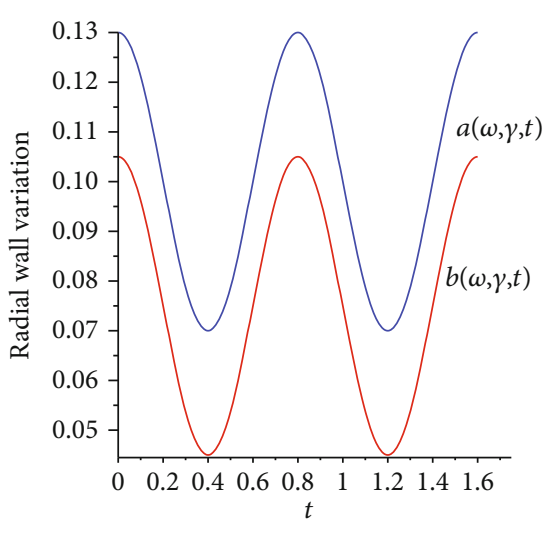

(a)

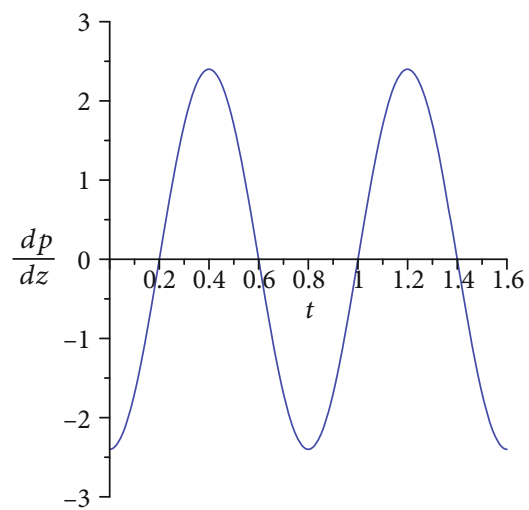

(b)

FIGURE 1: Variations of radial wall and pressure gradient $d p / d z$.

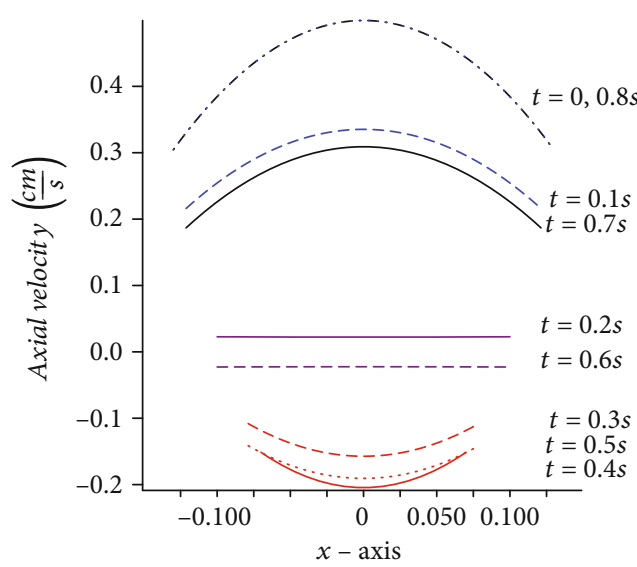

(a)

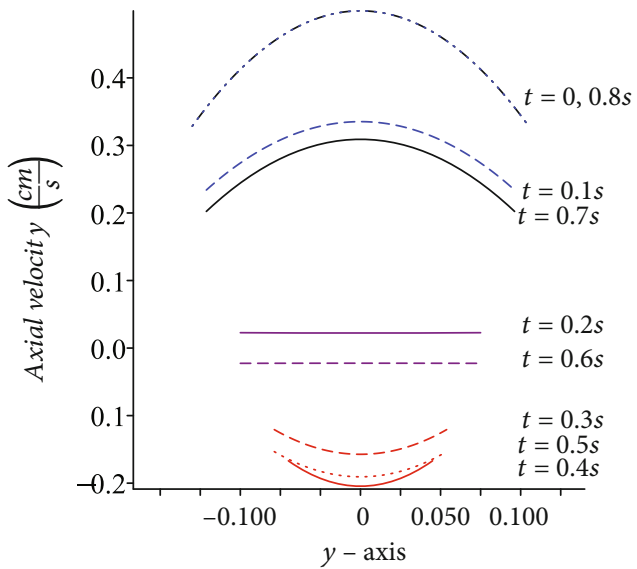

(b)

FIGURE 2: Variation of axial velocity in the first full wave cycle at various times along the radial axes $x$ and $y$ for the oscillation amplitude of $0.03 \mathrm{~cm}$ and the slip length of 0.05 .

Figure 2 illustrates the axial velocity in the first full wave cycle for $a_{0}=0.1 \mathrm{~cm}, a_{0}=0.075 \mathrm{~cm}$, oscillation amplitude $\gamma=0.3 \mathrm{~cm}$, frequency $\omega=5 \pi / 2$, and a slip length $\beta=0.05$. The velocity decreases during periods of wall expansion, $t \in[0,0.2)$, and wall contraction, $t \in[0.4,0.6)$, but increases during periods of wall expansion, $t \in(0.6,0.8]$ and wall contraction, $t \in(0.2,0.4]$. Effect of oscillatory wall motion and compliance on flow pattern have been observed in flow channel.

To examine the effect of oscillation wall and slip length on the temperature field, we plot the temperature profiles and its contour on the cross-section $z=5 \mathrm{~cm}$ at the $30^{\text {th }}$ wave cycle. In Figure 3, it is observed that temperature varies between 0.1 and $1.05^{\circ} \mathrm{C}$ at the $30^{\text {th }}$ wave cycle for the slip length of 0.05 and oscillation amplitude of $0.03 \mathrm{~cm}$.

As the frequency increases, the wall responds by oscillating with increasing amplitude. To investigate the impact of oscillation amplitude, $\gamma$, on the velocity and temperature field, we choose the values of $\gamma$ between 0.01 and $0.05 \mathrm{~cm}$ and select the investigated point at the center of the elliptical cross-section $(x, y)=(0,0)$. The results indicate that during the expansion period as shown in Figure 4(a), there is a linear relationship between oscillation amplitude and velocity $u(0$, $0, t)$ as the increase of oscillation amplitude rises the velocity and there is an increase of the velocity during the contraction period but the fluid moves in the opposite direction as shown in Figure 4(b). It is noted that an increase of oscillation amplitude rises the highest level of flow pressure gradient and thus enhances fluid flow rate. The obtained results are in well agreement with others $[7,14]$.

Figure 5 demonstrates the influence of slip length on the temperature field. Three values of the slip length $\beta$ including $0.005,0.05$, and 0.07 are chosen for the investigation. The results show that the temperature increases as the slip length decreases. It is further to mention that the rise in the slip length causes the fluid flow to move faster. This induces the observed decrease in blood temperature. These results agree with the results obtained by [18]-[20].

Furthermore, we analyse the effect of oscillation amplitude and slip length on the average temperature field by 

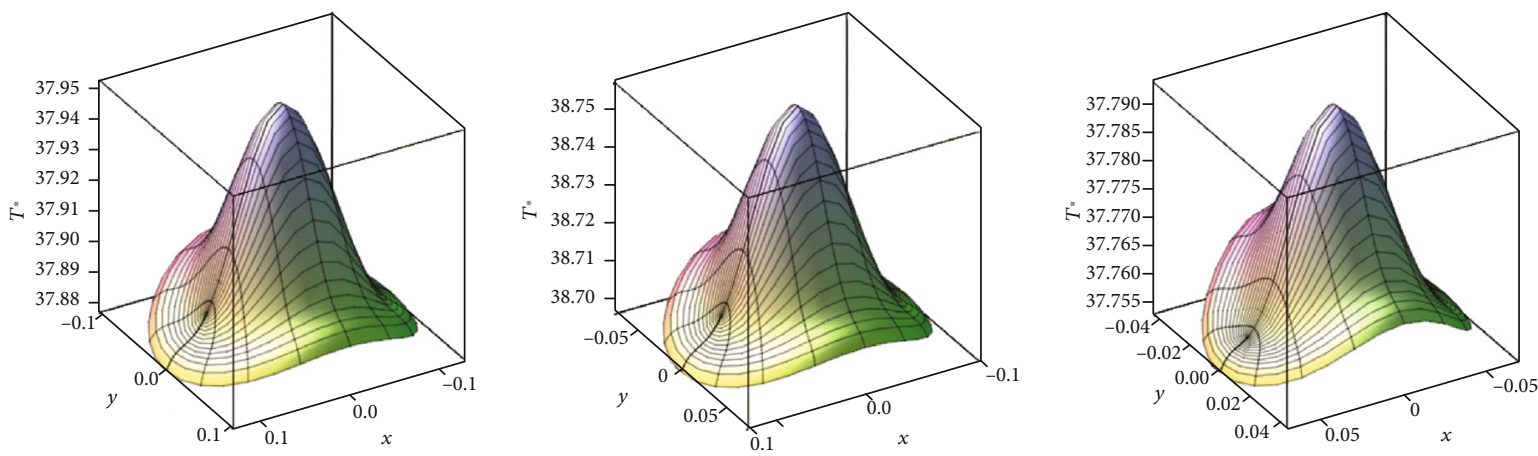

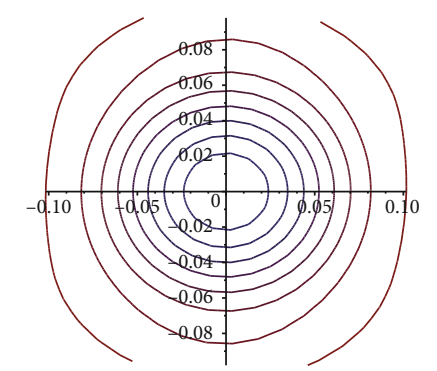

(a) $d p / d z=-2.4, t=24 \mathrm{~s}$

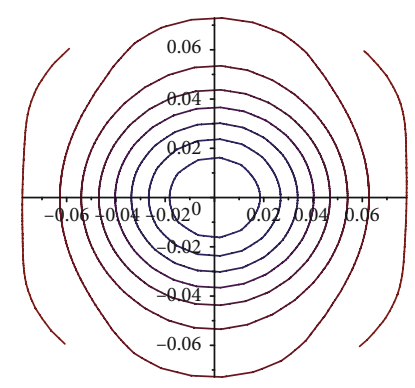

(b) $d p / d z=0, t=24.2 \mathrm{~s}$

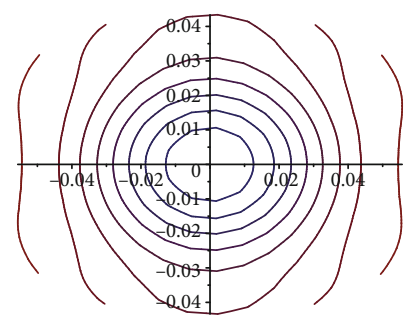

(c) $d p / d z=-2.4, t=24.4 \mathrm{~s}$

Figure 3: Temperature field at the $30^{\text {th }}$ wave cycle at three pressure gradients and different times for oscillation amplitude of $0.03 \mathrm{~cm}$ and slip length of 0.05 .
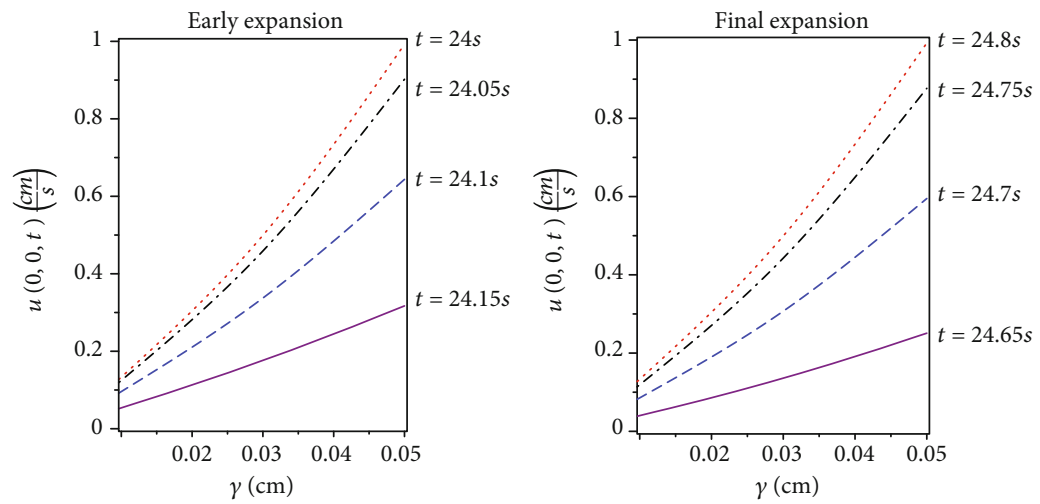

(a) Expansion period
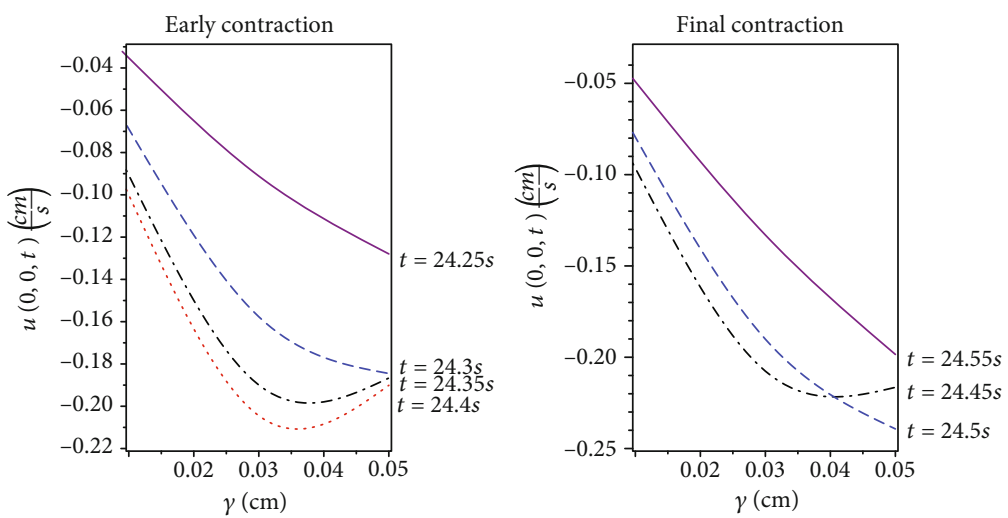

(b) Contraction period

FIGURE 4: The effect of $\gamma$ on the velocity $u(0,0, t)$ obtained from the model with a slip length of 0.05 at the $30^{\text {th }}$ wave cycle during two wall conditions: (a) wall expansion; (b) wall contraction. 

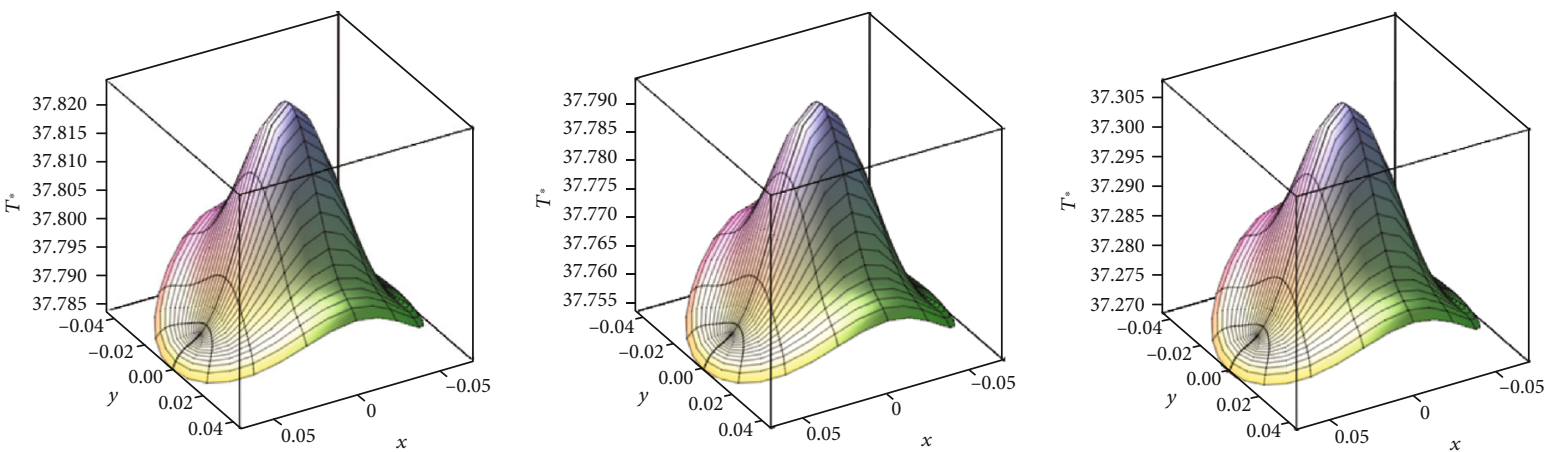

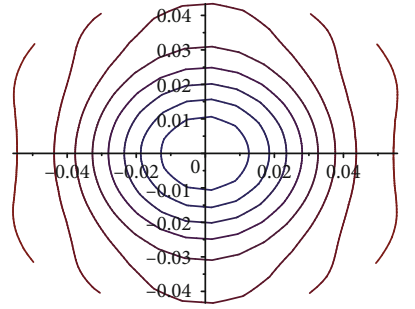

(a)

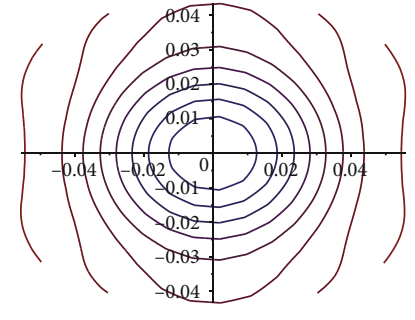

(b)

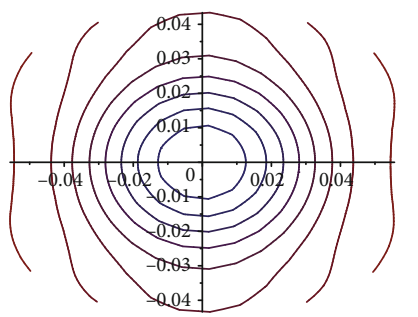

(c)

FIgURE 5: Temperature field at the $30^{\text {th }}$ wave cycle in the case of the highest positive pressure gradient $d p / d z=2.4$ for three different slip lengths: (a) $\beta=0.005$; (b) $\beta=0.05$; (c) $\beta=0.07$.

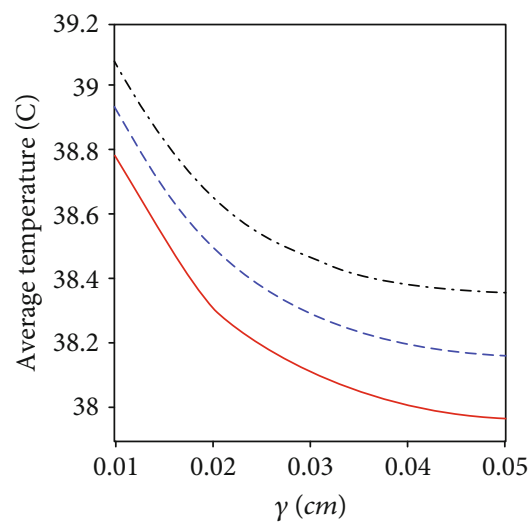

(a)

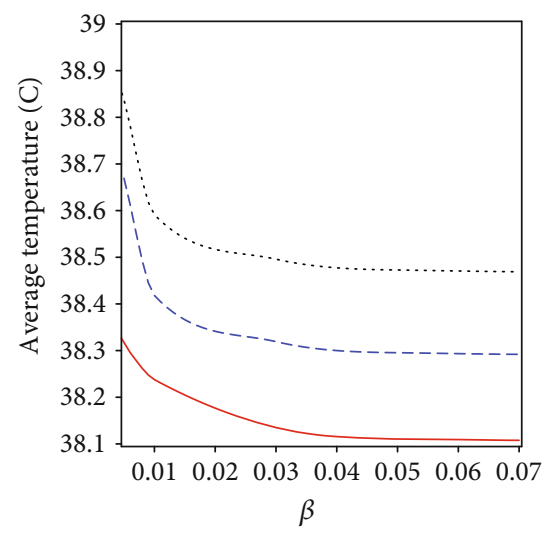

(b)

Figure 6: The effect of $\gamma$ and $\beta$ on the average temperature on the elliptic cross-section at three wave cycles.

varying $\gamma$ between 0.01 and $0.05 \mathrm{~cm}$ and $\beta$ between 0.005 and 0.07 at three different wave cycles including the $30^{\text {th }}, 40^{\text {th }}$, and $50^{\text {th }}$ cycles. It is found that the average temperature is inversely proportional to the oscillation amplitude and the slip length as shown in Figures 6(a) and 6(b). In addition, it is observed that the average temperature is higher in the higher wave cycle.

\section{Conclusions}

This paper studies the pressure-driven flow of fluid and heat transfer in an elliptical microchannel with radial oscillatory wall. Based on the assumption that there is no swirling flow, wall oscillation and pressure gradient are prescribed, and slip condition and heat convection are applied on the boundary; we proposed a mathematical model to investigate the effect of wall oscillation and slip length on the velocity and temperature field in the coronary artery. The results indicate that the slip length has a significant effect on blood flow and heat transfer during thermotherapy treatment. The wall oscillation also has a primary influence on the blood flow pattern and temperature distribution in the flow channel.

\section{Data Availability}

The data used to support the findings of this study are included within the supplementary material. 


\section{Conflicts of Interest}

The authors declare that there is no conflict of interest regarding the publication of this paper.

\section{Acknowledgments}

The first author would like to acknowledge the full support from the Science Achievement Scholarship of Thailand (SAST) and also the partial support from the Faculty of Graduate Studies, Mahidol University, Salaya, Phuttamonthon, Nakhonpathom Province, 73170, Thailand.

\section{Supplementary Materials}

One supplementary material is, a Maple code, available at https://github.com/Nattawan1992/Thermal-Slip-Flow/ issues/1. (Supplementary Materials)

\section{References}

[1] C. D. Bertram, "Experimental studies of collapsible tubes," in Flow Past Highly Compliant Boundaries and in Collapsible Tubes, P. W. Carpenter and T. J. Pedley, Eds., pp. 51-65, Springer, Dordrecht, 2003.

[2] P. Ricco, "Modification of near-wall turbulence due to spanwise wall oscillations," Journal of Turbulence, vol. 5, p. 20, 2004.

[3] M. Quadrio and S. Sibilla, "Numerical simulation of turbulent flow in a pipe oscillating around its axis," Journal of Fluid Mechanics, vol. 424, pp. 217-241, 2000.

[4] C. Xu and W. X. Huang, "Transient response of Reynolds stress transport to spanwise wall oscillation in a turbulent channel flow," Physics of Fluids, vol. 17, no. 1, article 018101, 2005.

[5] A. M. Thomas, G. K. Thich, and R. Narayanan, "Low Reynolds number flow in a channel with oscillating wavy-walls: an analytical study," Chemical Engineering Science, vol. 61, no. 18, pp. 6047-6056, 2006.

[6] R. J. Whittaker, M. Heil, and S. L. Waters, "The energetics of flow through a rapidly oscillating tube with slowly varying amplitude," Philosophical Transactions of the Royal Society A: Mathematical, Physical and Engineering Sciences, vol. 369, no. 1947, pp. 2989-3006, 2011.

[7] L. Espìn and D. T. Papageorgiou, "Viscous pressure-driven flows and their stability in channels with vertically oscillating walls," Physics of Fluids, vol. 24, no. 2, article 023604, 2012.

[8] S. P. Shupti, M. M. Molla, and M. Mia, "Pulsatile nonNewtonian fluid flows in a model aneurysm with oscillating wall," Frontiers in Mechanical Engineering, vol. 3, p. 12, 2017.

[9] M. G. Blyth, P. Hall, and D. T. Papageorgiou, "Chaotic flows in pulsating cylindrical tubes: a class of exact NavierStokes solutions," Journal of Fluid Mechanics, vol. 481, pp. 187-213, 2003.

[10] D. Mateescu and D. A. Venditti, "Unsteady confined viscous flows with oscillating walls and multiple separation regions over a downstream-facing step," Journal of Fluids and Structures, vol. 15, no. 8, pp. 1187-1205, 2001.

[11] I. Yudhistira and M. Skote, "Direct numerical simulation of a turbulent boundary layer over an oscillating wall," Journal of Turbulence, vol. 12, no. 9, 2011.
[12] B. J. Gireesha, M. Umeshaiah, B. C. Prasannakumara, N. S. Shashikumar, and M. Archana, "Impact of nonlinear thermal radiation on magnetohydrodynamic three dimensional boundary layer flow of Jeffrey nanofluid over a nonlinearly permeable stretching sheet," Physica A: Statistical Mechanics and its Applications, vol. 549, article 124051, 2020.

[13] S. O. Adesanya and O. D. Makinde, "MHD oscillatory slip flow and heat transfer in a channel filled with porous media," $U P B$ Scientific Bulletin, Series A: Applied Mathematics and Physics, vol. 76, pp. 197-204, 2014.

[14] J. C. Umuvathi, A. J. Chamkha, A. Mateen, and A. Al-Mudhaf, "Unsteady oscillatory flow and heat transfer in a horizontal composite porous medium channel," Nonlinear Analysis: Modelling and Control, vol. 14, no. 3, pp. 397-415, 2009.

[15] B. K. Jha and A. O. Ajibade, "Free convective flow between vertical porous plates with periodic heat input," ZAMM, vol. 90, no. 3, pp. 185-193, 2010.

[16] B. K. Jha and A. O. Ajibade, "Effect of viscous dissipation on natural convection flow between vertical parallel plates with time-periodic boundary conditions," Communications in Nonlinear Science and Numerical Simulation, vol. 17, no. 4, pp. 1576-1587, 2012.

[17] A. A. Hakeem and K. Sathiyanathan, "An analytic solution of an oscillatory flow through a porous medium with radiation effect," Nonlinear Analysis: Hybrid Systems, vol. 3, no. 3, pp. 288-295, 2009.

[18] A. M. Sobh, "Peristaltic slip flow of a viscoelastic fluid with heat and mass transfer in a tube," Mathematical Problems in Engineering, vol. 2012, Article ID 815747, 23 pages, 2012.

[19] B. Wiwatanapataphee, W. Sawangtong, N. Khajohnsaksumeth, and Y. H. Wu, "Oscillating pressure-driven slip flow and heat transfer through an elliptical microchannel," Advances in Difference Equations, vol. 2019, no. 1, 2019.

[20] S. T. Mohyud-Din, U. Khan, N. Ahmed, and W. Sikander, "A study of velocity and temperature slip effects on flow of water based nanofluids in converging and diverging channels," International Journal of Applied and Computational Mathematics, vol. 1, no. 4, pp. 569-587, 2015.

[21] C. Y. Wang, "Ritz method for slip flow in super-elliptic ducts," European Journal of Mechanics-B/Fluids, vol. 43, pp. 85-89, 2014.

[22] B. K. Raut, V. N. Patil, and G. Cherian, "Coronary artery dimensions in normal Indians," Indian Heart Journal, vol. 69 , no. 4 , pp. 512-514, 2017. 